auf die physiologischen Vorgänge, die bei der Expektoration cine Rolle spielen, zu studieren. Die Physiologie lehrt, daß für die Herausbeförderung pathologischer Sekretansammlungen in den Luftwegen die Flimmerepithelien, die mit Ausnahme der Alveolen und der kleinsten Bronchiolen die gesamte Bronchialschleimhaut auskleiden, von Bedeutung sind. Die von Rudolf Virchow (vgl. Engelman n in He rman $\mathrm{n}$ s Handbuch der Physiol. Bd. 1, T. I, S. 402, 1879) im Jahre 1854 an der menschlichen Trachea beobachtete Anregung der Flimmertätigkeit bei direkter Applikation von Natron- nnd Kalilauge kommt für die arzneiliche Behandlung kaum in Betracht. Später beobachtete Eng elma n n; daß kleine Mengen Kohlensäure und Ammoniak die Tätigkeit der Flimmerepithelien anregen, größere eine Lähmung hervorrufen. Diese Beobachtung kann eher für die Beurteilung der Wirkung einiger Expektorantien herangezogen werden. Für die Expektoration ist weiter die Tätigkeit der glatten Muskulatur der Lunge maßgebend, ihr Tonus wird vom Nervus vagus und vom Nervus sympathicus beherrscht. Es ist allerdings fraglich, ob man durch arzneiliche Beeinflussung der Lungenmuskulatur eine Expektoration hervorrufen kann. AuBer den genannten mehr mechanischen Faktoren für das Zustandekommen der Expektoration ist von wesentlicher Bedeutung der physikalische Zustand des in den Luftwegen vorhandenen pathologischen Sekrets. Es liegt ja auf der Hand, daß ein dünnflüssiges Sekret durch die Tätigkeit der Flimmerzellen leichter herausbefördert wird als ein zähes, dickflüssiges Sekret. Wir kennen nun eine ganze Reihe von Mitteln, die auf die physikalische Beschaffenheit des Lungensekrets einen EinfluB ausüben. Zunächst Salze. Das für den Körper wichtigste Salz, das Kochsalz, kann als Prototyp dieser Wirkung betrachtet werden. Es verläßt den Körper nicht nur durch die Nieren, sondern wird auch auf Schleimhäuten ausgeschieden. Gelangt nun Kochsalz zur Ausscheidung, so muls auch Wasser ausgeschieden werden, und darauf beruht offenbar seine schleimauflösende Wirkung. Wir können daher eher von einem Solvens als von einem Expektorans im engeren Sinne sprechen. Diese Wirkung kommt nicht nur dem Kochsalz, sondern auch anderen Salzen zu. Bei den Ammoniumsalzen (Ammonium chloratum) kommt wahrscheinlich die oben von Engelmann gefundene Anregung der Flimmertätigkeit durch Ammoniak in Betracht. A m monium chlorid ist bekanntlich Bestandteil der bekannten Mixtura solvens, die außer diesem Salz noch Succus liquiritiae enthält. Auch der Liquor ammonii anisatus wird häufig verordnet. Bei den Karbonaten und Bikarbonaten des Kaliums und Natriums haben wir auBer der Salzwirkung noch eine spezifische A!:aliwirkung, die besonders bei der Auflösung des Schleims von Bedeutung ist, und Mineralwässer, wie das Emser und andere, die außer Kochsalz Natriumbikarbonat enthalten, spielen bei der Behandlung von chronischen Bronchitiden eine bedeutende Rolle. Sie werden nicht nur getrunken, sondern auch in geeigneten Inhalatorien zerstäubt inhaliert.

Eine weitere Gruppe von Expektorantien bilden die Emetika. Diese Mittel rufen durch Vermittlung des Brechzentrums in der Medulla oblongata Erbrechen hervor. In den ersten Stadien dieser Wirkung haben wir neben den anderen Symptomen der Nausea Zunahme der Sekretion aller Drüsen, wobei die Bronchialdrüsen ebenfalls zur gesteigerten Sekretion angeregt werden. Dadurch geht das dickflüssige, zähe Sekret in dünnflüssiges über und kann durch die Tätigkeit der Flimmerepithelien leicht herausbefördert werden. Apomorphin, Radix Ipecacuanhae, Brechweinstein (Tartarus stibiatus) und der Goldschwefel (Stibium sulfuratum aurantiacum) sind die wichtigsten Mittel dieser Gruppe. Die lpekakuanhawurzel stammt aus Brasilien. Sie gehört zur Zeit zu den teuersten Arzneimitteln (1 g kostet $3,10 \mathrm{M}$.). Man hat neuerdings als Ersatz dieser Droge die Veilchenwurzel (Viola odorata) vorgeschlagen. Diese einheimische Pflanze ist ja sehr leicht zu beschaffen. Ob ihre Wirkungen mit denen der Ipekakuanha vollkommen übereinstimmen, muß noch näher geprüft werden.

Am wenigsten sind wir orientiert über eine Klasse von Ex-

Aus dem Pharmakologischen Institut der Universität in Berlin. (Direktor: Geh.-Rat Heffter.)

\section{Ueber Expektorantia.}

\section{Von Prof. Dr. G. Joachimogitu.}

Die experimentelle Pharmakologie versucht bei allen Arzneimitteln, die nach empirischer Erfahrung eine Wirkung auf den kranken Menschen ausüben, eine Grundlage für ihre therapeutische Anwendung durch das Experiment zu geben. Vielfach gelingt es nicht, dieses Ziel zu erreichen. Es wäre nicht richtig, in solchen Fällen dem Praktiker den Rat zu geben, von der Anwendung solcher Arzneistoffe abzusehen. Wir versuchen bei diesen Arzneimitteln, so gut es geht, die experimentellen Resultate mit den klinischen Erfahrungen in Einklang zu bringen. Die experimentellen Grundlagen für die Anwendung der Expektorantien, mit denen wir uns hier ganz kurz beschäftigen wollen, ist zur Zeit recht dürftig. Zum Teil liegt dies daran, daß wir keine exakten Methoden haben, um am lebenden Menschen oder an lebenden Tieren die Wirkung von einverleibten Mitteln

1) Zschr. f. Konstitutionslebre 2. Abt. 8, S. 42-53; M. K1. 1921 Nr. 11 S. 321 f. ) D Zschr. f. Nervhlk. 65, IS. 138. - a) Enuresis in Pfaundler.Schlossmann Handb. d. Kindhlk. - 4) D. Zschr. f. Nervhlk. 74, S. 343 . pektorantien, die aus verschiedenen Ptlanzen stammen. Ihre wirksamen Stoffe nennt man Saponine, ohne diesen Begriff chemisch genau definieren zu können. Sie kommen als Glykoside vor, d. h. bei ihrer Spaltung entstehen Zuckerarten. Die Bezeichnung Saponin haben sie èrhalten, weil ihre Lösungen wie Seifenwasser schäumen. Mit Blutkörperchen im Reagenzglas in Berührung gebracht, rufen sie noch bei sehr schwachen Konzentrationen Hämolyse hervor. Die gleiche Wirkung wird beobachtet bei direkter Injektion in die Blutbahn eines lebenden Tieres. Theoretisch interessant ist, daß in Gegenwart von Cholesterin eine hämolytische Wirkung nicht eintritt. Wir sind also ziemlich genau über die Wirkung der Saponine auf das Blut unterrichtet, uni so mangelhafter sind unsere Kenntnisse über die expektorierende Wirkung der saponinhaltigen Drogen. Auffallend ist, daß während die Zahl der saponinhaltigen Pflanzen ungemein groB ist, nur wenige als Expektorantien Anwendung gefunden haben. Bei Berücksichtigung dieser Tatsache könnte es beinahe zweifelhaft erscheinen, ob es lediglich auf den Saponingehalt ankommt. Innerlich können saponinhaltige Drogen in großen Dosen gegeben werden. Sie reizen die Schleimlaäute und können Speichelfluß und reflektorisch vermehrte Sekretion der Bronchialdrüsen, Uebelkeit und Erbrechen, ähnlich wie Ipekakuanha hervorrufen. Die aus Nordamerika stammende Radix Senegae, die in Südamerika einheimische Cortex Quillaiae und die aus SüdruBland und dem Orient importierte Radix Liquiritiae sind die wichtigsten Mittel dieser Gruppe, Durch die 
Valutaverhältnisse ist ihre Verordnung wie die der Ipekakuanhawurzel recht kostspielig geworden. Als Ersatz der Senegawurzel ist die einheimische Primelwurzel (Primula officinalis, Primula elatior, Primula vulgaris) vorgeschlagen worden. $\mathrm{Ob}$ es sich hier um eine gleich wertige Ersatzdroge handelt, kann, da experimentell die Frage kaum zu lösen ist, nur durch genaue klinische Beobachtung entschieden werden. Die Frage gehört also in das Gebiet der klinischen Pharmakologie, deren Wichtigkeit nur wenigen Klinikern klar geworden-ist Sie ist ebenso wichtig wie die Diagnostik und wird in Zukunft die Bedeutung der Pharmakologie für die praktische Medizin erhöhen. Die Species pectorales des Deutschen Arzneibuchs, die Eibischwurzel (Radix Althaeae), Süßholz (Radix Liquiritiae), Veilchenwurzel (Rhizoma ridis), Huflattichblätter (Folia Farfarae), Wollblumen (Flores verbasci) und Anisöl enthalten, sind ein billiges Ersatzmittel der Senegawurzel, da, abgesehen von dem Preisunterschied, der Patient das Infus (1. EBlöffel auf 2 bis 3 Tassen Wasser) selbst bereiten kann.

Ein weiteres Expektorans ist die Benzoësäure (Acidum benzoicum, $\mathrm{C}_{6} \mathrm{H}_{5} \mathrm{COOH}$ ). Sie soll nach den Vorschriften des Deutschen Arzneibuchs aus der aus Siam stammenden Benzoè gewonnen werden. Es ist gar keine Frage, daß die synthetische Benzoếsäure die gleichen Wirkungen hat, und es empfiehit sich, das synthetische Präparat $z u$ verordnen ( $1 \mathrm{~g}$ Acidum benzoicum kostet $1,30 \mathrm{M}$., $1 \mathrm{~g}$ Acidum benzoicum syntheticum aber nur $0,50 \mathrm{M}$.). Endlich sei das aus Terpentinöl stammende Terpinum hydratum erwähnt. Auch dieses Präparat wird bei Bronchitiden vielfach mit gutem Erfolg angewandt. Terpentinöl wurde vor dem Kriege zum größten Teil aus Amerika und Frankreich importiert. Man hat nicht daran gedacht, die inländischen Koniferen durch eine rationelle Harzung zur Terpentingewinnung heranzuziehen. Die Notlage während und nach dem Kriege hat jedoch gelehrt, dal man auch das inländische Produkt verwerten kann. Terpentinöl ist besonders wichtig, weil bekanntlich daraus der synthetische Kampfer gewonnen wird. 